\title{
Managing Type 2 Diabetes and Binge Eating Disorder: A Qualitative Study of Patients' Perceptions and Lived Experiences
}

Meg G. Salvia ( $\square$ msalvia@g.harvard.edu )

Boston University https://orcid.org/0000-0002-0428-443X

Marilyn D. Ritholz

Joslin Diabetes Center

Katherine L.E. Craigen

Walden Behavioral Care

Paula A. Quatromoni

Boston University

Research article

Keywords: binge eating disorder, type 2 diabetes, prediabetes, qualitative research, eating disorder treatment

Posted Date: February 10th, 2022

DOl: https://doi.org/10.21203/rs.3.rs-944659/v1

License: (c) (i) This work is licensed under a Creative Commons Attribution 4.0 International License. Read Full License 


\section{Abstract \\ Background}

The overlap in prevalence between type 2 diabetes (T2DM) and binge eating disorder (BED) is substantial, with adverse physical and mental health consequences. Little is known about patients' efforts at managing these two conditions simultaneously. The research objective was to explore patients' experiences managing co-existing T2DM/prediabetes and BED.

\section{Methods}

Qualitative descriptive study using semi-structured interviews. Participants included 21 women with T2DM or prediabetes ( $90 \%$ non-Hispanic White; mean age $49 \pm 14.8$ years, mean BMI $43.8 \pm 8.4 ; 48 \%$ with T2DM and mean $\mathrm{HbA} 1 \mathrm{c}$ was $8.4 \%$ ). Interviews were analyzed using thematic analysis and NVivo software.

\section{Results}

Qualitative analysis revealed that participants reported binge episodes frequently started in childhood or adolescence and went undiagnosed for decades; notably, they recalled that diabetes diagnosis preceded BED diagnosis. They also described trying to lose weight throughout their lives and how feelings of deprivation, shame, and failure exacerbated binge eating. Participants further reported how binge eating made diabetes self-care and outcomes worse. Finally, participants observed that when BED treatment and diabetes management were synergistically integrated, they experienced improvements in both binge eating and glycemic outcomes. This integration included reframing negative thoughts surrounding BED and diabetes self-management and increasing their understanding of how the two disorders were connected.

\section{Conclusions}

Findings highlight the importance of increasing healthcare providers' awareness of and screening for BED in the treatment of diabetes and for developing specific integrated interventions that address both diagnoses.

\section{Introduction}

The prevalence and public health challenges associated with type 2 diabetes (T2DM) and prediabetes are well-known (1). Less well understood is the extent to which binge eating disorder (BED) occurs alongside T2DM and prediabetes and how it affects treatment recommendations and outcomes. BED, added as a stand-alone diagnosis in the Diagnostic and Statistical Manual, 5th edition in 2013 (2), is characterized 
by recurrent binge episodes in which an individual eats an objectively large amount of food in a discrete time with associated hallmark feelings of loss of control, distress, guilt, and shame but without compensatory purging behaviors. BED is prevalent in about $2-3 \%$ of the general population (3).

Estimates of the prevalence of eating disorders among patients with T2DM vary; studies suggest 7-20\% of patients with T2DM have binge eating behaviors (4-10), with $8 \%$ having a clinical diagnosis of BED (10). Among those with BED, approximately one-third have T2DM (8). Binge eating negatively impacts glycemic control $(11,12)$, but it is unclear how patients manage these two conditions or what specific challenges they face. There is mixed evidence regarding the directionality of the association between binge eating and T2DM. Individuals with diabetes are at increased risk of disordered eating behaviors (8, 12); and the prescribed or perceived rigidity of diet and weight loss recommendations for prevention and management of T2DM can exacerbate disordered eating among those with or predisposed to eating disorders (13-15). Studies have also found an association between disordered eating and incident metabolic syndrome and T2DM (16), and youth with disordered eating behaviors had lower insulin sensitivity compared with those who exhibited normal eating patterns (17).

For individuals who experience both T2DM/prediabetes and BED, conflicting recommendations in medical and nutritional management present daily challenges. For example, diabetes prevention and management recommendations typically encourage weight-loss efforts to support insulin sensitivity and delay disease progression. However, BED treatment recommendations often encourage calorically adequate, balanced, and varied eating patterns with intentional therapeutic exposure to specific foods to reduce the impact that hunger and dietary restraint play in binge episodes.

The aim of this study was to qualitatively explore the experiences and perspectives of adults concurrently managing T2DM/prediabetes and BED to increase healthcare providers' understanding of patients' challenges and to inform the development of practical treatment strategies for this population.

\section{Methods}

\section{Sample}

Adult participants were recruited from an eating disorder treatment center in Massachusetts that offered intensive outpatient programming specifically for BED. Medical records from admissions between 2015 and 2019 were screened for cooccurring T2DM. Because binge eating has been associated with metabolic syndrome (18), we also recruited those with prediabetes (defined as "prediabetes," "metabolic syndrome" or "impaired glucose tolerance" noted in patients' medical records). Additional inclusion criteria required completion of $>2$ weeks of the treatment program, discharge at least six months prior to study enrollment, and the ability to participate in an English-spoken interview. Exclusion criteria consisted of cognitive impairment or severe psychopathology limiting the ability to recall program participation or engage in the interview, or discontinuing the intensive outpatient program to admit to a higher level of care. 
Participants attended one study visit at the clinic. All participants gave informed consent before beginning and received monetary compensation $(\$ 50)$ for their participation.

\section{Data Collection}

In-person interviews were conducted with the use of a semi-structured interview guide outlining core research questions and follow-up prompts. Interviews were audio-recorded and transcribed verbatim. Blinded weights were obtained at the study visit using the clinic's calibrated scale. Biomedical data was obtained by self-report at the time of the interview and from medical records from participants' outpatient providers. Participants gave consent separately to retrospectively request medical records from their outpatient providers. The questionnaire version of the Eating Disorder Examination (EDE-Q), a measure designed to assess the range and severity of problematic eating behaviors and attitudes (19-21), was self-administered along with a brief survey to collect demographic data and diabetes management information.

\section{Data Analysis}

Interview transcripts were coded and analyzed by the multidisciplinary research team including PAQ (epidemiologist and registered dietitian), MDR (licensed psychologist with expertise in diabetes and qualitative research), MGS (registered dietitian with expertise in BED and T2DM), and KLEC (licensed psychologist with expertise in BED). This ensured investigator triangulation and supported the internal validity of the data (22). Codes were inductively identified, and a codebook was developed and iteratively refined until consensus was attained and data saturation reached for each theme. NVivo software was used to manage data and facilitate the analysis (23).

\section{Results}

\section{Sample characteristics}

In our sample of 319 BED patients, $22 \%$ had either T2DM $(n=25)$ or prediabetes $(n=45)$. Of these, 8 did not meet further eligibility criteria, 10 declined, and 31 did not respond. Twenty-one participants were interviewed; of these, all were women. Participant characteristics are shown in Table 1.

Table 1. Participant characteristics ( $\mathrm{n}=21$ women) 


\begin{tabular}{|c|c|c|}
\hline & Mean (range) & $\mathbf{N}(\%)$ \\
\hline \multicolumn{3}{|l|}{ Participant age, in years } \\
\hline Mean (range) & $49(19-66)$ & \\
\hline \multicolumn{3}{|l|}{ Participant race } \\
\hline White & & $19(90)$ \\
\hline Black or African American & & $1(5)$ \\
\hline Multiple race identities indicated & & $1(5)$ \\
\hline \multicolumn{3}{|l|}{ BMI } \\
\hline Mean (range) & $43.8(30.2-63.9)$ & \\
\hline $30.0-39.9$ & & $7(33)$ \\
\hline $40.0-49.9$ & & $10(48)$ \\
\hline $50.0-59.9$ & & $3(14)$ \\
\hline $60.0-69.9$ & & $1(5)$ \\
\hline \multicolumn{3}{|l|}{ Education history } \\
\hline Completed high school & & $1(5)$ \\
\hline Some college or technical school & & $6(29)$ \\
\hline College degree & & $11(52)$ \\
\hline Graduate degree & & $3(14)$ \\
\hline \multicolumn{3}{|l|}{ Employment status } \\
\hline Employed, full-time & & $10(48)$ \\
\hline Employed, part-time & & $5(24)$ \\
\hline Disabled, employed part-time & & $1(5)$ \\
\hline Disabled, not able to work & & $4(19)$ \\
\hline Student & & $3(14)$ \\
\hline \multicolumn{3}{|l|}{ Insurance plan (at time of interview) } \\
\hline Medicaid or Medicare & & $5(24)$ \\
\hline Group/private insurance through employer & & $13(62)$ \\
\hline Individual private insurance (self-purchased) & & $3(14)$ \\
\hline Treatment team composition (at time of inten & & \\
\hline
\end{tabular}




\begin{tabular}{|lc|} 
Primary care (physician or nurse practitioner) & $21(100)$ \\
\hline Therapist & $11(52)$ \\
\hline Endotitian & $4(19)$ \\
\hline Diabetes educator & $7(33)$ \\
\hline Psychiatrist & $1(5)$ \\
\hline
\end{tabular}

Ten participants had T2DM diagnoses and 11 had prediabetes, as shown in Table 2. Almost three quarters of participants reported binge eating onset in childhood or adolescence. Persistence of binge behavior is noted with varying degrees of frequency in this population that received treatment for BED. EDE-Q data do not suggest high clinical concern of the eating disorder at the time of interview; on average, all mean scores were $<4$. However, some individual scores were elevated, and weight and shape concern subscales trended the highest.

Table 2. Participants' diabetes and binge eating outcomes $(n=21)$ 


\begin{tabular}{|c|c|c|}
\hline Characteristic & Mean (range) & $\mathbf{N}(\%)$ \\
\hline \multicolumn{3}{|l|}{ Diabetes diagnosis } \\
\hline Type 2 diabetes & & $10(48)$ \\
\hline Current hemoglobin A1c (\%) & $8.4(6.4-14.0)$ & \\
\hline Diabetes duration (years) & $12(5-18)$ & \\
\hline Prediabetes & & $11(52)$ \\
\hline Years since prediabetes diagnosis & $5.7(1-19)$ & \\
\hline Number progressing to T2DM diagnosis & & $1(9)$ \\
\hline \multicolumn{3}{|l|}{ Age/Life stage of binge onset (self-reported) } \\
\hline Childhood & & $11(52)$ \\
\hline Adolescence & & $4(19)$ \\
\hline Young adulthood & & $4(19)$ \\
\hline Middle adulthood & & $2(10)$ \\
\hline \multicolumn{3}{|l|}{$\begin{array}{l}\text { Frequency of binge eating episodes } \\
\text { (at time of interview) }\end{array}$} \\
\hline$<1 \mathrm{x} /$ month & & $5(24)$ \\
\hline $1-2 x /$ month & & $2(10)$ \\
\hline $1 \mathrm{x} /$ week & & $4(19)$ \\
\hline $2-3 x /$ week & & $3(14)$ \\
\hline $4-6 x /$ week & & $2(10)$ \\
\hline $1 \mathrm{x} /$ day & & $3(14)$ \\
\hline$>1 x /$ day & & $2(10)$ \\
\hline \multicolumn{3}{|l|}{ EDE-Q scores* ${ }^{*}$} \\
\hline Global Score & $2.6(0.6-5.3)$ & \\
\hline Restraint & $1.5(0-3.6)$ & \\
\hline Eating concern & $2.5(0-6)$ & \\
\hline Shape concern & $3.1(0.3-6)$ & \\
\hline Weight concern & $3.5(0.5-6)$ & \\
\hline
\end{tabular}


*EDE-Q global and subscale scores range from 0-6; higher number indicates more problematic eating behaviors and attitudes; a cut-off of 4 on the global score generally represents clinical significance (24).

\section{Qualitative Themes}

We identified four central themes in our qualitative analysis: 1) Early Onset and Delay in Diagnosis of Binge Eating, 2) Dieting Drives Binge Patterns; 3) Bidirectional Impact of DM and BED; and 4) Need for Synergy in Treatment Recommendations.

\section{Early Onset and Delay in Diagnosis of Binge Eating}

Study participants almost universally reported that binge eating behaviors preceded the T2DM/prediabetes diagnosis, often by several decades. Many reported binge eating in childhood or adolescence:

I mean, I've binged since I was a kid. I'm not sure I had an official diagnosis that I was aware of, but I was aware that I binged.

- Participant \#5, age 66, T2DM

Only two participants described BED developing later in life and cited mid-life changes and increased loneliness as factors in binge eating onset:

It wasn't really until my 50s, when my kids were gone out of the house, and I was on my own, and I didn't have anybody there. I had a very busy lifestyle raising three kids. And that, I think, kept me from bingeing and giving in to food because they always filled up my life.

- Participant \#12, age 66, T2DM

Most participants also described delays in getting diagnosed with BED, spending much of those early decades without treatment. Many participants reported they were only diagnosed with BED when they chose to disclose behaviors to their medical team or therapists with the goal of seeking admission to a BED treatment program, which they'd often identified on their own from an internet search. In most cases, receiving the BED diagnosis was not as scary to participants as the diabetes diagnosis was; it was a source of relief and validation, opening up the possibility of treatment.

\section{Dieting Drives Binge Patterns}

Participants frequently mentioned longstanding efforts at dieting or limiting intake of food (particularly sources of sugar and other carbohydrates) in their weight-loss efforts. This occurred prior to and concurrent with diabetes diagnoses. Participants described oscillating between windows of controlling intake with windows of binge episodes. Some described feeling like their efforts at dieting or food restriction were related to increased binge eating. Participants also described early-life dieting as 
contributing to a sense of deprivation, followed by the guilt or shame associated with binge eating. These experiences contributed to repeating cycles of restrict-binge-restrict:

The weight definitely influenced the binge eating disorder because I would do everything, all my eating and all my exercise was to the goal of losing weight to look thin. ... I would eat so many veggies and so many low-carb, low-fat, whatever... and later I would binge and feel bad about it, and those foods that I do like felt like demons.

- Participant \#17, age 22, prediabetes

I thought about what led to my binge eating because that's not a behavior I would ever have thought I would have. And I think, I really do think that the restriction is what made me start to binge.

- Participant \#10, age 57, prediabetes

Weight loss was also a priority articulated in health-care discussions with providers and family members. Weight-loss surgery was recommended for most participants; for those who underwent surgeries in the years preceding their attendance in the eating disorder treatment program, loss-of-control eating continued post-surgery and weight was regained.

\section{Bidirectional Impact of DM and BED}

\section{Binge eating behaviors were responsible for development of diabetes}

Many participants felt that binge eating directly contributed to their diabetes and prediabetes; they endorsed feelings of guilt and shame around the diagnosis associated with fears that they "did this to themselves" because of disordered eating behaviors.

\section{Heterogeneous impact of diabetes on binge eating}

Participants with diabetes described the diagnosis experience as scary and prompted new realizations for the health implications of binge eating. Some described increased but ultimately unsustainable efforts to control binge episodes in the wake of the diabetes diagnosis:

The stakes were higher [with diabetes diagnosis], and when I failed it was more devastating. When I was first diagnosed [with diabetes] I was scared, and I would go through periods of being scared and controlling my eating really well and thereby controlling my blood sugars really well. And the problem was it didn't last more than 2, 3, maybe 4 months. And then it regressed.

- Participant \#13, age 59, T2DM

Others, however, experienced increases in binge episodes in response to the stress of the diagnosis. Participants with prediabetes expressed less concern about the way binge eating impacted their current 
glycemia or future T2DM risk.

\section{Binge eating made diabetes outcomes worse}

Participants described binge episodes negatively impacting diabetes management, causing chronically elevated blood glucose levels that were difficult to bring down to goal ranges. Most participants described how difficult it was to interrupt binge cycles or reduce binge behaviors on their own even though they valued improving diabetes outcomes:

It disables me. The binge eating is the crux of it all. Like, if I could just stop that, managing diabetes would not be a problem. But because I have such an unhealthy attachment to food, I can't stop. You know, I can't stop because I'm gonna die, because I'm gonna lose my feet, because I'm gonna lose my sight... it still isn't enough, and that's pretty pathetic.

- Participant \#11, age 56, T2DM

It was very clear to me that those [BED] behaviors were making my diabetes and numbers worse. And it felt like this awful conundrum, that I need to treat the diabetes, but I can't treat the diabetes until I deal with the eating, and the eating is making the diabetes worse. It felt like a diabolical combination. The two things were just intertwined and interlocked, and each was making the other worse.

- Participant \#13, age 59, T2DM

Self-care and diabetes management decreased in response to binges

Most participants who self-monitored blood glucose described skipping glucose checks after bingeing as a means of avoiding information, or out of feelings of shame. They reported not wanting to have elevated numbers provide visible evidence of the binges to themselves or when showing their glucose logs to their diabetes treatment teams:

You don't want to look at that [high glucose] number because you know it was your fault. You did it, and you could've avoided it... I would be afraid to look at the number because it would confirm that I'm a failure.

- Participant \#18, age 63, T2DM

In terms of medication and insulin, participants endorsed adherence, reporting taking oral medications and insulin as prescribed. Notably, some described estimating an insulin dose that might cover the binge episode despite not knowing the carbohydrate content of the binge:

If I'm going to be on a binge, I'm going to be way above 350. So I feel like I'm pretty safe at a 20 [unit] dose. Which is not what my - my endocrinologist would just flip out if she heard me say that because that's not at all a responsible, healthy way to maintain your diabetes. And I do realize that, but it's better than doing nothing. 


\section{Binge eating treatment improved diabetes self-care}

Many participants reported treatment increased awareness of how BED and diabetes were connected. They reported learning how meal timing, nutritional content of foods, and binge patterns impacted their blood glucose levels. Some reported seeing improvements in diabetes control, such as fewer glycemic excursions and more consistent T2DM self-care, as binge behaviors decreased:

Managing the binge eating disorder helped me manage the prediabetes, which had taken more of the backburner... I really have been making a lot of progress. I binge so much less than before. I restrict so much less than before.

- Participant \#17, age 22, prediabetes

\section{Need for Synergy in Treatment Recommendations}

Participants described experiencing conflict when they attempted to integrate differing treatment recommendations, resulting in frustration and confusion:

I was always going back and forth with, you know, working on trying to heal the eating disorder but then also freaking out: 'but I have diabetes, I need to get serious about this, I need to buckle down, like, I'm doing damage to myself.'

- Participant \#1, age 40, T2DM

For example, some participants described a lack of cohesion when incorporating foods that were typically viewed as "off-limits" from the diabetes-management lens in the context of BED treatment. Specifically, the inclusion of carbohydrate-based foods into the meal plan designed to normalize eating patterns concerned some individuals and challenged treatment buy-in. Participants also experienced conflict when diabetes recommendations focused on caloric restriction for weight loss while BED treatment prioritized reducing or extinguishing bingeing, taking a weight-neutral approach. Some described these circumstances as stressful and contributed to a decreased sense of well-being, particularly regarding diabetes self-care and psychosocial stress.

In contrast, some participants described a process of treatment synergy occurring when providers explicitly accounted for both BED and diabetes diagnoses in treatment discussions and recommendations, resulting in self-reported improvement in glycemia. They described how first and foremost providers needed to be aware of both diagnoses and that when BED was kept secret and not openly disclosed, medical providers did not inquire about symptoms or incorporate BED into the treatment plan:

I think part of my denial before treatment was not getting the connection between my eating and my blood sugar levels. 
Similarly, participants noted how some BED providers had limited knowledge of diabetes care, compromising their ability to integrate diabetes-management goals.

Finally, participants stated that the integrated discussion of both diagnoses helped address the negative thoughts, self-judgment and self-blame that contributed to both binge eating and poor diabetes management:

I think they [BED and T2DM recommendations] were in sync about forgiving yourself, being kinder to yourself, giving you permission to fail and rebound... Because I do believe it's the emotional beating yourself up that starts that vicious, vicious cycle of binge eating and feeling like a failure ... I'm more consistent with [blood glucose] monitoring because, again, I try to tell myself it's really just a number. It's like a road map... rather than have it be a judgment. And it helps me to go in a different direction.

- Participant \#18, age 63, T2DM

\section{Discussion}

In this qualitative study, we explored 21 adult women's experiences of living with and managing T2DM/prediabetes and BED. We found almost all participants reported that binge eating preceded their diagnosis of diabetes. They also voiced that their longstanding efforts at weight loss contributed to binge patterns and believed bingeing subsequently led to developing diabetes. They further stated that binge eating made diabetes self-care more difficult. While they universally took diabetes medication as prescribed, many reported avoiding checking blood glucose levels to conceal the visibility of binge episodes for themselves and from their diabetes treatment team. Participants described the need for BED treatment and diabetes management to be synergistically integrated, and when this occurred, participants reported improvements in both binge eating and glycemic outcomes. Helpful treatment strategies included increasing understanding of how the two conditions were connected, reframing negative thoughts surrounding BED and diabetes self-management while building appropriate behavioral and coping skills. To achieve this, screening and non-judgmental discussion of BED by medical providers, and referral to multidisciplinary eating disorder treatment professionals, appear essential.

As in other studies $(8,15)$, we found that binge eating often preceded the diabetes diagnosis. However, most of our participants reported that the BED diagnosis did not occur until after they were first diagnosed with diabetes. One contributor to the delay in BED diagnosis may be the lack of familiarity with and assessment for BED among healthcare providers $(25,26)$. Our finding underscores the importance of primary care and diabetes professionals' screening for BED as early as possible. Using existing screening tools such as the BEDS-7 (27) can help identify these patients. In the setting of T2DM, similar to what has been observed in type 1 diabetes (28), it may be helpful to ask a targeted question when patterns such as avoiding checking blood glucose levels are observed. For example, one might engage discussion by asking, "Can you tell me more about your eating habits on the days when you find 
yourself not doing glucose checks?" Studies have reported that individuals who experienced binge episodes were significantly younger at age of T2DM diagnosis compared to those without binge eating episodes (29). Importantly, younger age of T2DM diagnosis increases the risk for negative cardiovascular and mortality outcomes (30). Collectively, these findings highlight the critical need for routine screening and early intervention for people with BED and T2DM $(4,31-33)$.

BED has been demonstrated to be a risk factor for the development of T2DM $(34,35)$. Previous studies found binge eating high-fat and high-calorie foods contributed to decreased insulin sensitivity (36), higher fasting blood glucose levels, and increased insulin resistance (28). Previous research in a population of women with T2DM (37) suggested the temporal order of BED and diabetes is that binge eating preceded both dieting onset and T2DM diagnosis. Our participants' timelines followed this general pattern except they tended to report dieting preceded and may have precipitated the onset of binge eating. This difference in findings should be further explored to better understand the temporal order of bingeing, dieting and diabetes.

Participants also perceived BED as interfering with their diabetes management by contributing to decreased self-care behaviors. Avoidance of post-binge glucose monitoring was rooted in self-blame and shame around binges. These feelings are salient contributors to patients' inability or reluctance to selfdisclose binge eating behavior and/or to ask providers for help. When healthcare professionals consider these seemingly non-adherent behaviors and approach them with curiosity and an awareness of the underlying shame and self-blame, they may foster useful conversations that invite patients to open up about their struggles. Importantly, participants reported adhering to prescribed oral medications and insulin doses. Thus, unlike women with type 1 diabetes and eating disorders who use insulin omission as a calorie-purging technique (38), our participants did not omit or manipulate medication for weight-loss purposes. This finding needs to be investigated further.

Upon getting treatment for BED, most women reported reduced or extinguished binge eating behaviors. This pattern was sustained post-treatment for some, but other participants continued to struggle with binge eating recurrence, particularly around life changes and significant stressors. This suggests that for many, BED may be a more chronic condition and that cycles of binge eating are a concern deserving ongoing evaluation in primary care settings. Because some women reported binge eating onset or relapse around significant mid-life changes, screening for BED can have important clinical relevance when women face circumstances such as divorce, death of a spouse, experiencing an "empty nest" or retirement.

Participants also voiced concerns about the conflict they experienced between BED and diabetes treatment recommendations. Although nutritional recommendations for T2DM have moved from strict prescriptions for carbohydrates, protein and fat to more individualized recommendations based on the assessment of patients' eating patterns and personal preferences including culture, tradition, religion, access, health beliefs and personal goals (39), weight loss is still a major focus of treatment for T2DM. Treatment of patients with BED and diabetes may require not only more flexible eating patterns and 
advice, but also an understanding that the eating disorder needs to be treated first, before weight loss is considered and evaluated for its therapeutic relevance (4). Further, as with recommendations for eating disorders and T1DM (38), relaxing the glucose targets for diabetes treatment may allow patients to engage more effectively in eating disorder treatment (4). The finding that participants in our study reported improvements in glycemic control and reduced binge episodes with BED treatment is a desirable outcome, showing dual benefits of eating disorder treatment on both diagnoses. This observation warrants further study with larger and more diverse samples of persons diagnosed with BED and T2DM/prediabetes. More needs to be understood about why some participants sustain these improvements as well as how BED treatment may influence the progression of prediabetes to type 2 diabetes.

There is agreement among diabetes and eating disorder professional organizations that a multidisciplinary treatment team is the optimal approach for BED and diabetes $(4,39)$. Ideally, the team includes a primary care medical provider and/or endocrinologist, mental health provider or psychologist, dietitian, diabetes educator, and sometimes a psychiatrist. This collaborative multi-disciplinary approach allows for an integration of diabetes and BED treatments so patients experience knowledgeable and unified care. Of note, participants voiced that adequate expertise in and consideration for both BED and diabetes was helpful, when it existed. Some, but not all, participants reported having an integrated experience and described the absence of conflict in their treatment recommendations; this allowed them to better understand how BED and diabetes were connected.

There are several limitations to this study. The sample consisting of mostly non-Hispanic White welleducated women who were treated at a specialized eating disorder clinic limits the generalizability of the findings. The prevalence of BED among Black women and White men is like that among White women (40) and the prevalence of T2DM in minorities is far greater than in non-Hispanic Whites (1). Thus, research on diabetes and BED needs to include more diverse populations. Because we studied a treated population, we do not know about the experiences of adults who live with BED and T2DM who do not seek treatment. This caveat again emphasizes the important role that primary care providers have in screening for BED and directing patients to eating disorder treatment. Otherwise, the burden of awareness of appropriate, integrated treatment messages falls too heavily to the primary care team without the added assistance from eating disorder professionals. Finally, research also needs to investigate health care providers' perspectives to better inform treatment strategies for co-occurring BED and T2DM/prediabetes. In addition, self-reported improvements in health outcomes were documented in this study, and larger quantitative studies are needed to confirm these observations.

In summary, participants with BED and diabetes described struggling with weight loss for many years and how they perceived this struggle as contributing to their binge eating and development of diabetes. They also voiced concerns about how binge eating made diabetes management more difficult and how conflicting messages about the management of BED and diabetes were frustrating. Importantly, participants benefitted from treatment approaches that explained the connection between the two disorders and provided evidence of how improving one disorder contributed to improvement of the other. 
These clinical suggestions highlight how healthcare providers can approach the treatment of BED and $\mathrm{T} 2 \mathrm{DM} /$ prediabetes by incorporating screening, referrals, and effective patient communication and education strategies.

\section{Abbreviations}

T2DM

type 2 diabetes

BED

binge eating disorder

EDE-Q

Eating Disorder Examination questionnaire

\section{Declarations}

Ethics approval and consent to participate: Study procedures were approved by the Boston University Institutional Review Board. All study participants gave their informed consent.

Consent for publication: Not applicable

Availability of data and materials: The dataset used and analysed during the current study are not publically available due to the sensitivity of the data (i.e., transcribed interviews from human subjects) and are available from the corresponding author upon reasonable request.

Competing Interests: KLEC is an employee of Walden Behavioral Care, and PAQ is a Senior Consultant to Walden Behavioral Care's Department of Nutrition.

Funding: This study was funded by the Dudley Allen Sargent grant out of Boston University, which did not influence the study's design, conduct, or reporting.

Authors contributions: MGS, PAQ, and MDR contributed to the research design, MGS and PAQ were involved in data collection, and all authors participated in the data analysis and manuscript development. All authors read and approved the final manuscript.

\section{References}

1. Centers for Disease Control and Prevention. National Diabetes Statistics Report. 2020. Atlanta GA. Centers for Disease Control and Prevention, U.S. Dept of Health and Human Services; 2020.

2. American Psychiatric Association. (2013). Diagnostic and statistical manual of mental disorders (5th ed.). Arlington, VA.

3. Kessler RC, Berglund PA, Chiu WT, et al. The prevalence and correlates of binge eating disorder in the World Health Organization world mental health surveys. Biol Psychiatry. 2013;73(9):904-14. 
4. García-Mayor RV, García-Soidán FJ. Eating disorders in type 2 diabetic people: brief review. Diabetes Metab Syndr. 2017;11:221-4.

5. Papelbaum M, Appolinário JC, Moreira RO, Ellinger VC, Kupfer R, Coutinho WF. Prevalence of eating disorders and psychiatric comorbidity in a clinical sample of type 2 diabetes mellitus patients. Braz $\mathrm{J}$ Psychiatr. 2005;27:135-8.

6. Herpertz S, Albus $\mathrm{C}$, Wagener R, Kocnar M, et al. Comorbidity of diabetes and eating disorders. Does diabetes control reflect disturbed eating behavior? Diabetes Care. 1998;21(7):1110-6.

7. Thornton LM, Watson HJ, Jangmo A, et al. Binge-eating disorder in the Swedish national registers: Somatic comorbidity. Int J Eat Disord. 2017;50(1):58-65.

8. Raevuori A, Suokas J, Haukka J, et al. Highly increased risk of type 2 diabetes in patients with binge eating disorder and bulimia nervosa. Int J Eat Disord. 2015;48:555-62.

9. Santana D, Mitchison D, Gonzalez-Chica D, et al. Associations between self-reported diabetes mellitus, disordered eating behaviours, weight/shape overvaluation, and health-related quality of life. Journal of Eating Disorders. 2019;7(1):35.

10. Abbott S, Dindol N, Tahrani AA, Piya MK. Binge eating disorder and night eating syndrome in adults with type 2 diabetes: a systematic review. J Eat Disord. 2018;6:36.

11. Meneghini LF, Spadola J, Florez H. Prevalence and associations of binge eating disorder in a multiethnic population with type 2 diabetes. Diabetes Care. 2006;29(12):2760-0.

12. Nicolau J, Simo R, Sanchis P, et al. "ED are Frequent Among T2DM Patients and Associated with Worse Metabolic Outcomes." Acta Diabetol 2015.

13. Criego A, Crow S, Goebel-Fabbri AE, Kendall D, Parkin C. Eating disorders and diabetes: screening and detection. Diabetes Spectr. 2009;22(3):143-6.

14. Young-Hyman DL, Davis CL. Disordered eating behavior in individuals with diabetes: importance of context, evaluation, and classification. Diabetes Care. 2010;33(3):683-9.

15. Gagnon C, Aime A, Belanger C. Predictors of Comorbid Eating Disorders and Diabetes in People with Type 1 and Type 2 Diabetes. Canadian Journal of Diabetes. 2017;41(1):52-7.

16. Yoon C, Jacobs DR, Duprez DA. Problematic eating behaivors and attitudes predict long-term incident metabolic syndrome and diabetes: The Coronary Artery Risk Development in Young Adults Study. Int J Eat Disord. 2019;52(3):304-8.

17. Nip ASY, Reboussin BA, Dabelea D, et al. Disordered Eating Behaviors in Youth and Young Adults with Type 1 or Type 2 Diabetes Receiving Insulin Therapy: The SEARCH for Diabetes in Youth Study. Diabetes Care. 2019;42(5):859-66.

18. Wassenaar E, Friedman J, Mehler PS. Medical Complications of Binge Eating Disorder. Psychiatr Clin North Am. 2019;42(2):275-86.

19. Berg KC, Peterson CB, Frazier P, Crow SJ. Psychometric evaluation of the eating disorder examination and Eating Disorder Examination-Questionnaire: A systematic review of the literature. Int $\mathrm{J}$ Eating Disorders. 2011;45:428-38. 
20. Luce $\mathrm{KH}$, Crowther $\mathrm{JH}$. The reliability of the Eating Disorder Examination - Self Report Questionnaire Version (EDE-Q). Int J Eating Disorders. 1999;25:349-51.

21. Reas DL, Grilo CM, Masheb RM. Reliability of the eating disorder examination-questionnaire in patients with binge eating disorder. Behav Res Ther. 2006;44:43-51.

22. Miles MB, Huberman AM. Qualitative Data Analysis: An Expanded Sourcebook. Thousand Oaks: Sage Publications; 1994.

23. QSR International Pty Ltd. (2018) NVivo (Version 12), https://www.qsinternational.com/nvivoqualitative-data-analysis-software/home.

24. Aardoom JJ, Dingemans AE, Slof Op't Landt. M. C. T., Van Furth EF Norms and discriminative validity of the Eating Disorder Examination Questionnaire (EDE-Q). Eating Behaviors. 2012; 13(4), 305-309.

25. Chevinsky JD, Wadden TA, Chao AM. Binge Eating Disorder in Patients with Type 2 Diabetes: Diagnostic and Management Challenges. Diabetes Metab Syndr Obes. 2020;13:1117-31.

26. Supina D, Herman BK, Frye CB, Shillington AC. Knowledge of binge eating disorder: a cross-sectional survey of physicians in the United States. Postgrad Med. 2016;128(3):311-6.

27. Herman BK, Deal LS, DiBenedetti DB, Nelson L, Fehnel SE, Brown TM. Development of the 7-Item Binge-Eating Disorder Screener (BEDS-7). Prim Care Companion CNS Disord. 2016;18(2):10.4088/PCC.15m01896.

28. Goebel-Fabbri AE, Fikkan J, Franko DL. Insulin restriction and associated morbidity and mortality in women with type 1 diabetes. Diabetes Care. 2008;31:415-9.

29. Abraham TM. Massaro JM, Hoffmann U, et al. Metabolic Characterization of Adults with Binge Eating in the General Population: the Framingham Heart Study. Obesity. 2014;22(11):2441-9.

30. Sattar N. Rawshani A, Franzen S, et al. Age at Diagnosis of Type 2 Diabetes Mellitus and Associations with Cardiovascular and Mortality Risks. Circulation. 2019;139(19):2228-37.

31. Lehman R. Krumholz HM. Tight Control of Blood Glucose in Long Standing Type 2 Diabetes. BMJ. 2009;338:b800.

32. Crow S. Kendall D, Praus B, et al. Binge Eating and Other Psychopathology in Patients with Type 2 Diabetes Mellitus. Int J Eating Disorders. 2001;30(2):222-6.

33. Harris SR. Carrillo M, Fujioka K. Binge-Eating Disorder and Type 2 Diabetes: A Review. Endocr Pract. 2021; 27: 158-64.

34. Nieto-Martinez R, Gonzalez-Rivas JP. Medina-Inojosa JR, et al. Are Eating Disorders Risk Factors for Type 2 Diabetes? A Systematic Review and Meta-analysis. Curr Diab Rep. 2017;17(12):138.

35. Hudson JI. Lalonde JK, Coit CE, et al. Longitudinal study of the diagnosis of components of the metabolic syndrome in individuals with binge-eating disorder. Am J Clin Nutr 2010;91:1568-73.

36. Parry SA. Woods RM, Hodson L, et al. A Single Day of Excessive Dietary Fat Intake Reduces WholeBody Insulin Sensitivity: The Metabolic Consequence of Binge Eating. Nutrients. 2017;9(8):818.

37. Kenardy J. Mensch M, Bowen K, et al. Disordered Eating Behaviours in Women with Type 2 Diabetes Mellitus. Eat Behav. 2001;2(2):183-92. 
38. Goebel-Fabbri AE. Diabetes and Eating Disorders. Journal of Diabetes Science and Technology. 2008.

39. Standards of Care in Diabetes-. 2021 Abridged for Primary Care Providers. Clinical Diabetes. 2021;39(1):14-43.

40. Smith DE, Marcus MD, Lewis CE, et al. Prevalence of Binge Eating Disorder, Obesity, and Depression in a Biracial Cohort of Young Adults. Ann Behav Med. 1998;20(30):227-32. 